\title{
Effect of breast cancer as the first or second primary cancer on the prognosis of women with thyroid cancer: a SEER database analysis
}

\author{
Jianglong Huang ${ }^{1 \#}$, Yihui Huang ${ }^{1 \#}$, Ling Zhou ${ }^{1}$, Sichao Chen ${ }^{1}$, Danyang Chen ${ }^{1}$, Wei Wei ${ }^{2}$, Chao Zhang ${ }^{3}$, \\ Min Wang ${ }^{1}$, Wei Zhou ${ }^{1}$, Wen Zeng ${ }^{4}$, Zeming Liu ${ }^{1}$, Liang Guo ${ }^{1}$ \\ ${ }^{1}$ Department of Plastic Surgery, Zhongnan Hospital of Wuhan University, Wuhan, China; ${ }^{2}$ Department of Pediatrics, St. John Hospital and Medical \\ Center, Detroit, MI, USA; ${ }^{3}$ Department of Cardiovascular Surgery, Union Hospital, Tongji Medical College, Huazhong University of Science and \\ Technology, Wuhan, China; ${ }^{4}$ Department of Ophthalmology, Zhongnan Hospital, Wuhan University, Wuhan, China \\ Contributions: (I) Conception and design: All authors; (II) Administrative support: M Wang, W Zhou, D Chen; (III) Provision of study materials or \\ patients: Z Liu; (IV) Collection and assembly of data: W Wei, C Zhang; (V) Data analysis and interpretation: J Huang, Y Huang; (VI) Manuscript \\ writing: All authors; (VII) Final approval of manuscript: All authors. \\ \#These authors contributed equally to this work. \\ Correspondence to: Liang Guo; Zeming Liu. Department of Plastic Surgery, Zhongnan Hospital of Wuhan University, Donghu Road 169, Wuchang \\ District, Wuhan, China. Email: guoliangwhzn@163.com; 6myt@163.com.
}

Background: Although many studies have evaluated the association between breast cancer (BC) and thyroid cancer (TC), this association has not been completely studied. In this study, we focused on the effect of $\mathrm{BC}$ as the first or second primary cancer on survival among women with TC. We present the following article in accordance with the STROBE reporting checklist.

Methods: Using the Surveillance, Epidemiology, and End Results (SEER) database, we performed a retrospective cohort analysis of 134,785 women with BC and TC between 1974 and 2016. The patients were divided into three subgroups, namely, TC only (TC group, n=128,888), BC after TC (TC2BC group, $\mathrm{n}=3,000$ ), and BC before TC (BC2TC group, $\mathrm{n}=2,897)$. The curves for overall survival (OS) and cancerspecific survival (CSS) were evaluated, and Cox regression analyses were performed to examine the effect of $\mathrm{BC}$ as the first and second primary cancer on the CSS and OS of TC patients.

Results: The CSS rates in the TC, TC2BC, and BC2TC groups were 96.96\%, 98.70\%, and 96.70\%, respectively, and the OS rates were $91.17 \%, 77.13 \%$, and $78.63 \%$, respectively. Using the TC group as a reference, the hazard ratios (HRs) for the CSS of the TC2BC and BC2TC groups were 0.333 [95\% confidence interval (CI): 0.243-0.456, $\mathrm{P}<0.001$ ] and 1.299 (95\% CI: 1.062-1.589, P=0.011), respectively. The corresponding HRs for the OS were 1.544 (95\% CI: 1.428-1.668, P<0.001) and 3.429 (95\% CI: 3.158-3.724, $\mathrm{P}<0.001)$, respectively.

Conclusions: Among the female patients with TC, BC, as the second primary cancer, has a significant protective effect on the CSS of TC patients; however, when BC is the first primary cancer, it is a risk factor. Besides, BC is a risk factor for the OS of TC patients, especially for patients who develop TC after BC.

Keywords: Breast neoplasms; second primary neoplasms; Surveillance, Epidemiology, and End Results (SEER); survival; thyroid neoplasms

Submitted Jun 02, 2020. Accepted for publication Sep 26, 2020.

doi: $10.21037 /$ tcr-20-2243

View this article at: http://dx.doi.org/10.21037/tcr-20-2243 


\section{Introduction}

The American Cancer Society (ACS) estimates that breast cancer (BC) will be the most common new cancer among women in 2020, accounting for $30 \%$ of new cancer diagnoses, and thyroid cancer (TC) will be the 5 th most common, accounting for $4 \%$ of new diagnoses (1). Many studies have extensively demonstrated the relationship between these two cancers in women (2-6). The overall prognosis of TC is relatively good, with 5 - and 10-year survival rates of $95-97 \%$ (5). However, due to the high incidence and favorable prognosis of TC, many survivors are at risk of developing a second primary cancer. A previous study reported that among the TC survivors, BC was the second primary cancer in $35 \%$ of the cases, and patients were at the highest risk of developing BC (7). The ACS previously estimated that there would be 276,480 new cases of BC among women in the United States in 2020, with 42,170 deaths from the disease (1). Early diagnosis and treatment improve the prognosis of $\mathrm{BC}$ patients. However, a previous study reported that women with BC had a significantly higher risk of developing a second primary cancer than the general population (8). Among the patients with BC, the incidence of TC was $0.28 \%$ (9).

Previous studies have evaluated the association between BC and TC $(2-6,10,11)$, and several studies have assessed the prognosis of $\mathrm{BC}$ as a second primary cancer in patients with TC. Cheng et al. found that a history of TC had a strong protective effect on BC-specific survival (12). Kuo et al. found that $\mathrm{BC}$ was less histologically aggressive in TC survivors (5). Other studies have also assessed the clinical characteristics of TC as the second primary cancer in patients with BC. Li et al. showed that the risk of TC development in patients with $\mathrm{BC}$ was higher than that in the general population (13), and Huang et al. showed that the BC survivors had a significantly higher risk of developing TC (10). Furthermore, Siołek et al. proposed that the aggregation of TC and BC might be related to the CHEK2 mutation (14); therefore, some people in the population will develop both BC and TC.

As mentioned above, most of the previous literatures discussed the influence of TC on BC, but few mentioned the influence of $\mathrm{BC}$ on TC. To further evaluate the relationship between $\mathrm{TC}$ and $\mathrm{BC}$ in women, we considered $\mathrm{BC}$ the influencing factor to assess its effect on the patients with TC. In the present study, we used case data from the Surveillance, Epidemiology, and End Results (SEER) database to investigate the effects of $\mathrm{BC}$ as the first or second primary cancer on survival among women with TC, considering the patients with only TC as the reference group. We present the following article in accordance with the STROBE reporting checklist (available at http://dx.doi. org/10.21037/tcr-20-2243).

\section{Methods}

The study was conducted in accordance with the Declaration of Helsinki (as revised in 2013). After signing the agreement and applying for a SEER account, we downloaded the SEER data software (SEER*Stat version 8.3.2) and case data. Since SEER is a publicly available database with anonymized data, ethical approval was not required. The study cohort comprised 134,785 women with TC who were registered in the SEER database (1974-2016). The patients were divided into the following three subgroups: TC only (TC group, n=128,888), BC after TC (TC2BC group, $n=3,000)$, and BC before TC (BC2TC group, $n=2,897)$.

The patients' demographic parameters were defined as race (white, black, and other or unknown) and age at diagnosis ( $\leq 55$ or $>55$ years). The year of diagnosis was classified two groups, namely, 1974-1994 and 1995-2016. The lesions were classified as solitary tumors, multifocal tumors, or unknown. Extension was classified as yes, no, or unknown. The treatment characteristics were defined as surgery (yes or no) and radiation (refused, yes, or unknown).

The patients' follow-up data were evaluated to calculate the rates of cancer-specific survival (CSS), overall survival (OS), and mortality rates per 1,000 person-years.

\section{Statistical analyses}

We used percentages to describe categorical variables, and the median and interquartile range (IQR) to describe continuous variables. Categorical variables were tested using Pearson's chi-square test. If the continuous variables were normally distributed, use the independent group test; if not, the Mann-Whitney test was used. For all tests, $P$ values $<0.05$ was considered statistically significant.

Univariate Cox regression analysis was performed to examine whether CSS and OS were associated with age, race, grade, histological type, TNM stage, extension status, radiation treatment, or surgery. Multivariate Cox regression analysis was performed in three steps. The first adjustment included age and race. The second adjustment included 
age, race, grade, histology, T-stage, $\mathrm{N}$-stage, $\mathrm{M}$-stage, multifocality and extension. Factors such as age, race, grade, histology, T-stage, N-stage, M-stage, multifocality and extension were included in the third adjustment. $\mathrm{P}$ value $<0.05$ was considered statistically significant.

Survival curves were generated using the Kaplan-Meier method and analyzed using the log-rank test. Statistical analyses were performed using SPSS software (version 23.0), StataSE software (version 5.1), and GraphPad Prism software (version 5.01). The differences were considered statistically significant for $\mathrm{P}$ values $<0.05$.

\section{Results}

\section{Differences in the demographic and clinicopathological characteristics of the TC, TC2BC, and BC2TC groups}

The demographic and clinicopathological characteristics of the TC, TC2BC, and BC2TC groups are displayed in Table 1. Patients aged $<55$ years accounted for $70.33 \%$ of the TC group, $58.07 \%$ of the TC2BC group, and $33.52 \%$ of the BC2TC group. The proportion of white people in the TC, TC2BC, and BC2TC groups was $81.08 \%, 81.79 \%$, and $80.63 \%$, respectively. The median survival of patients in the TC, TC2BC, and BC2TC groups was 22.00 (IQR, 13.00-49.00) months, 155.00 (IQR, 95.00-241.75) months, and 57.50 (IQR, 24.00-104.00) months, respectively. The CSS rates in the TC, TC2BC, and BC2TC groups were $96.96 \%, 98.70 \%$, and $96.70 \%$, respectively, and the corresponding OS rates were $91.17 \%, 77.13 \%$, and $78.63 \%$, respectively.

\section{Cancer-specific and all-cause mortality in the TC, TC2BC, and BC2TC groups}

The cancer-specific mortality and all-cause mortality in the TC, TC2BC, and BC2TC groups are shown in Table 2. The cancer-specific mortality rates in the TC, TC2BC, and BC2TC groups were $3.0 \%, 1.3 \%$, and $3.3 \%$, respectively, and the cancer-specific mortality rates per 1,000 person-years were 3.036 [95\% confidence interval (CI): 2.935-3.141], 0.868 (95\% CI: 0.634-1.188), and 4.869 (95\% CI: 3.941-6.015), respectively. The corresponding all-cause mortality rates for the TC, TC2BC, and BC2TC groups were $8.8 \%, 22.9 \%$, and $21.4 \%$, respectively, and the corresponding all-cause mortality rates per 1,000 personyears were 9.161 (95\% CI: 8.985-9.342), 15.264 (95\% CI: 14.164-16.450), and 32.555 (95\% CI: $30.000-35.327$ ), respectively.

\section{Comparison of CSS and OS among the TC, TC2BC, and BC2TC groups}

The results of the univariate analysis for the CSS and OS in the different groups are shown in Table 3. Using the TC group as the reference, the hazard ratios (HRs) for the CSS of the TC2BC and BC2TC groups were calculated to be 0.333 (95\% CI: $0.243-0.456, \mathrm{P}<0.001$ ) and 1.299 (95\% CI: $1.062-1.589, \mathrm{P}=0.011$ ), respectively, whereas the corresponding HRs for the OS were 1.544 (95\% CI: 1.428 1.668, $\mathrm{P}<0.001$ ) and 3.429 (95\% CI: $3.158-3.724, \mathrm{P}<0.001$ ), respectively.

The results of the multivariate analysis of the factors affecting the CSS and OS are shown in Table 4. In adjustment 1 , with the TC group as the reference, the HRs for the CSS and OS in the TC2BC group were 0.236 (95\% CI: $0.172-0.323, \mathrm{P}<0.001)$ and 1.146 (95\% CI: $1.06-1.238$, $\mathrm{P}=0.001$ ), respectively, whereas the HRs for the CSS and OS in the BC2TC group were 0.61 (95\% CI: 0.499-0.747, $\mathrm{P}<0.001)$ and 1.634 (95\% CI: $1.504-1.775, \mathrm{P}<0.001$ ), respectively. After adjustment 2, only the HR for the OS in the BC2TC group was significant with reference to the TC group (HR: 1.881, 95\% CI: 1.18-2.997, $\mathrm{P}<0.001$ ). After adjustment 3 , there was no significant difference in the HRs for either the CSS or OSS in the TC2BC group or the BC2TC group using the TC group as the reference.

The Kaplan-Meier curves of the CSS and OS for the TC, TC2BC, and BC2TC groups are shown in Figures 1,2, respectively. We found statistical differences in the CSS and OS among the three groups $(\mathrm{P}<0.05)$.

\section{Discussion}

Our study findings indicate that among women, the HR for the CSS of TC patients with BC as the second primary cancer was significantly reduced when the patients with only TC were considered the reference group, whereas the HR for the CSS of TC patients with BC as the first primary cancer was increased. Further, the HR for the OS in TC patients was significantly increased, regardless of whether BC was the first primary cancer or the second primary cancer. However, the HR of the OS increased more significantly when $\mathrm{BC}$ was the first primary cancer in TC patients than when $\mathrm{BC}$ was the second primary cancer. These results suggest that $\mathrm{BC}$, as the second primary 
Table 1 Clinicopathological characteristics of female patients with TC, TC2BC, and BC2TC groups

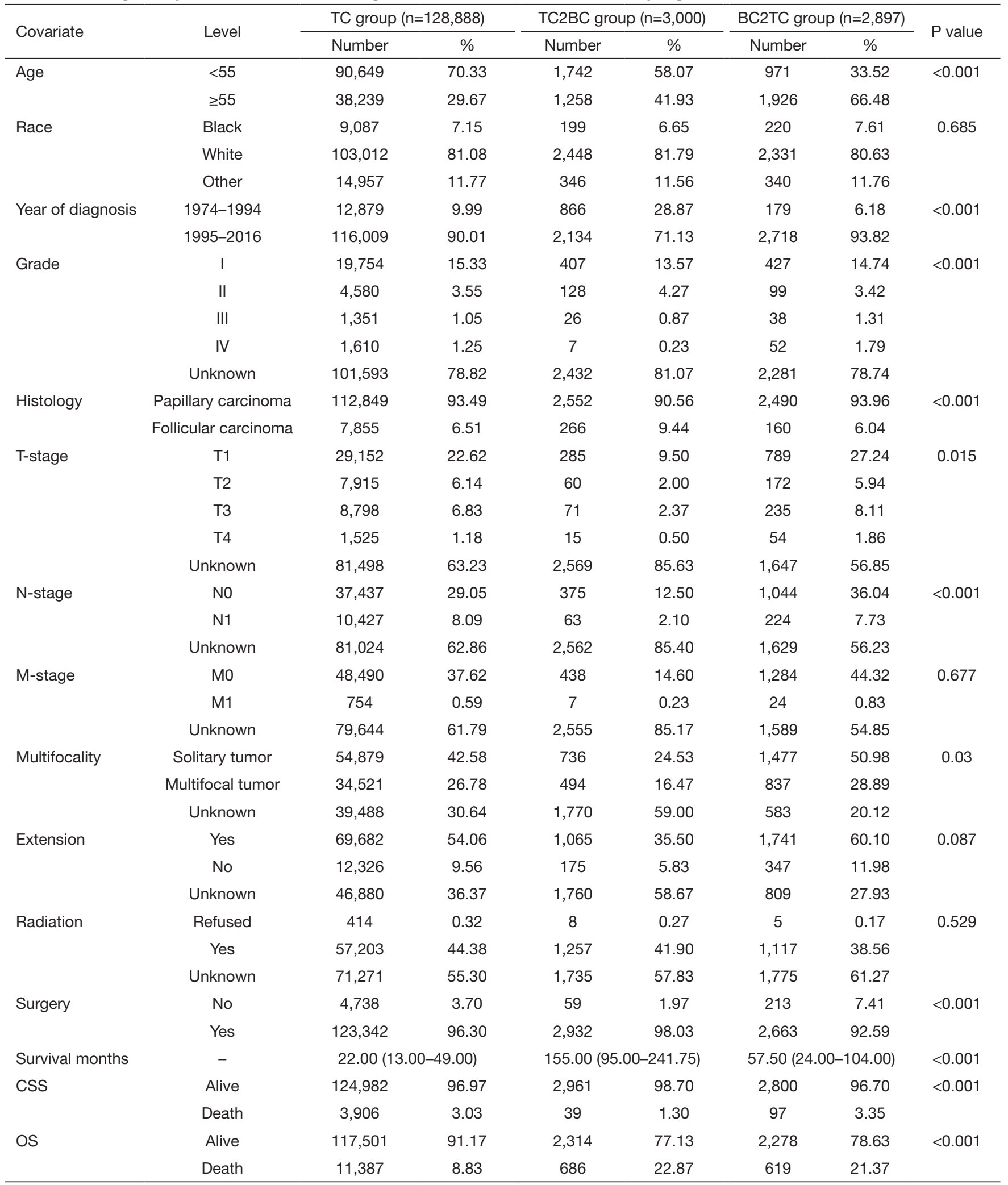

TC, thyroid cancer; BC, breast cancer; TC2BC, BC after TC; BC2TC, BC before TC; CSS, cancer-specific survival; OS, overall survival. 
Table 2 Cancer-specific mortality and all-cause mortality of TC, TC2BC, and BC2TC groups

\begin{tabular}{|c|c|c|c|c|c|c|c|c|}
\hline Group & \multicolumn{4}{|c|}{ Cancer-specific mortality } & \multicolumn{4}{|c|}{ All-cause mortality } \\
\hline $\mathrm{TC}$ & 3,906 & 3.0 & 3.036 & $2.935-3.141$ & 11,387 & 8.8 & 9.161 & $8.985-9.342$ \\
\hline TC2BC & 39 & 1.3 & 0.868 & $0.634-1.188$ & 686 & 22.9 & 15.264 & $14.164-16.450$ \\
\hline
\end{tabular}

TC, thyroid cancer; BC, breast cancer; TC2BC, BC after TC; BC2TC, BC before TC; Cl, confidence interval.

Table 3 Univariate analysis for CSS and OS in different groups

\begin{tabular}{|c|c|c|c|c|c|c|c|}
\hline Analysis & Group & \multicolumn{3}{|c|}{ CSS } & \multicolumn{3}{|c|}{ OS } \\
\hline \multirow[t]{3}{*}{ Analysis 1} & $\mathrm{TC}$ & Ref. & & & Ref. & & \\
\hline & TC2BC & 0.333 & $0.243-0.456$ & $<0.001$ & 1.544 & $1.428-1.668$ & $<0.001$ \\
\hline & BC2TC & 1.299 & $1.062-1.589$ & 0.011 & 3.429 & $3.158-3.724$ & $<0.001$ \\
\hline Analysis 2 & $\mathrm{TC} 2 \mathrm{BC}+\mathrm{BC} 2 \mathrm{TC}$ & 0.709 & $0.598-0.842$ & $<0.001$ & 2.083 & $1.966-2.207$ & $<0.001$ \\
\hline \multirow[t]{2}{*}{ Analysis 3} & TC2BC & Ref. & & & Ref. & & \\
\hline & ВC2TC & 4.762 & $3.222-7.038$ & $<0.001$ & 2.715 & $2.411-3.056$ & $<0.001$ \\
\hline
\end{tabular}

CSS, cancer-specific survival; OS, overall survival; HR, hazard ratio; Cl, confidence interval; TC, thyroid cancer; BC, breast cancer; TC2BC, BC after TC; BC2TC, BC before TC.

Table 4 Multivariate analysis for clinicopathologic factors that affect the CSS and the OS

\begin{tabular}{|c|c|c|c|c|c|c|c|}
\hline Analysis & Group & \multicolumn{3}{|c|}{ CSS } & \multicolumn{3}{|c|}{ OS } \\
\hline \multirow[t]{3}{*}{ Adjustment $1^{\mathrm{a}}$} & $\mathrm{TC}$ & Ref. & & & Ref. & & \\
\hline & TC2BC & 0.236 & $0.172-0.323$ & $<0.001$ & 1.146 & $1.06-1.238$ & 0.001 \\
\hline & BC2TC & 0.61 & $0.499-0.747$ & $<0.001$ & 1.634 & $1.504-1.775$ & $<0.001$ \\
\hline \multirow{2}{*}{ Adjustment $2^{\mathrm{b}}$} & TC2BC & 1.576 & $0.212-11.694$ & 0.656 & 0.866 & $0.274-2.731$ & 0.805 \\
\hline & ВС2TC & 0.443 & $0.108-1.821$ & 0.259 & 1.881 & $1.18-2.997$ & 0.008 \\
\hline \multirow[t]{2}{*}{ Adjustment $3^{c}$} & $\mathrm{TC}$ & Ref. & & & Ref. & & \\
\hline & TC2BC & - & - & 0.97 & 0.631 & $0.086-4.631$ & 0.651 \\
\hline
\end{tabular}

\footnotetext{
${ }^{a}$, Adjustment 1 for age and race; ${ }^{b}$, adjustment 2 for age, race, grade, histology, T-stage, N-stage, M-stage, multifocality and extension; ', adjustment 3 for age, race, grade, histology, T-stage, N-stage, M-stage, multifocality, extension, radiation and surgery. CSS, cancerspecific survival; OS, overall survival; $\mathrm{HR}$, hazard ratio; $\mathrm{Cl}$, confidence interval; $\mathrm{TC}$, thyroid cancer; $\mathrm{BC}$, breast cancer; $\mathrm{TC} 2 \mathrm{BC}$, $\mathrm{BC}$ after TC; BC2TC, BC before TC.
} 


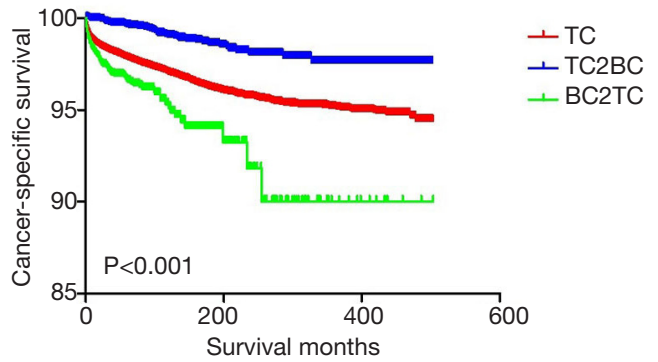

Figure 1 Kaplan-Meier curves of CSS for TC, TC2BC, and BC2TC groups. CSS, cancer-specific survival; TC, thyroid cancer; BC, breast cancer; TC2BC, BC after TC; BC2TC, BC before TC.

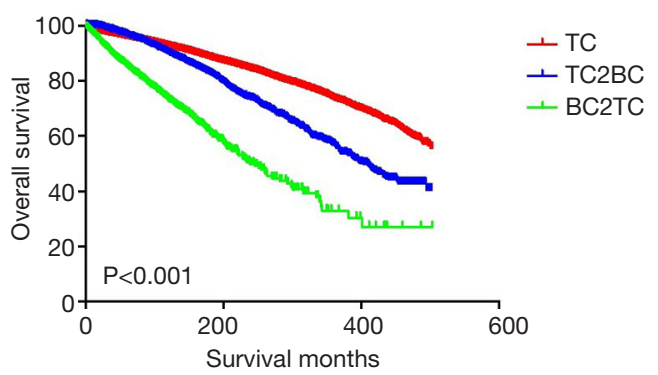

Figure 2 Kaplan-Meier curves of OS for TC, TC2BC, and BC2TC groups. OS, overall survival; TC, thyroid cancer; BC, breast cancer; TC2BC, BC after TC; BC2TC, BC before TC.

cancer, has a significant protective effect on the CSS in TC patients; however, $\mathrm{BC}$, as the first primary cancer, is a risk factor for the CSS in TC patients. In terms of the OS, BC is a risk factor for TC patients, and when $\mathrm{BC}$ is the first primary cancer, the risk of decreased OS is higher for TC patients.

Previous studies have observed a correlation between $\mathrm{BC}$ and TC $(2-6,10,11)$. This correlation is of great clinical significance for the $\mathrm{BC}$ and $\mathrm{TC}$ patients, as it may provide guidelines for the treatment of $\mathrm{BC}$ or $\mathrm{TC}$ as the second primary cancer, improve the prognosis of patients, and improve the survival rate. However, thus far, the effect of BC on TC has not been fully studied. Therefore, we selected the case data from the SEER database and considered the patients with only TC as the reference group to study the effect of $\mathrm{BC}$ as the first and second primary cancer on TC in women.

Marcheselli et al. showed that more than half of the $\mathrm{BC} 2 \mathrm{TC}$ patients were diagnosed with TC within 3 years of BC diagnosis (9), and Kuo et al. found that the median time to the diagnosis of $\mathrm{BC}$ in patients with $\mathrm{TC} 2 \mathrm{BC}$ was 5 years after diagnosis of TC (5). This difference in time to diagnosis may be related to the better prognosis of $\mathrm{TC}$ compared to that of BC. Furthermore, patients with BC are more likely to have thyroid-related examinations during the post-diagnosis follow-up observations, and minor thyroid lesions are likely to be detected in such follow-ups. In addition, low-dose L-thyroxine replacement therapy after TC may also be one of the reasons for the delayed time of $\mathrm{BC}$ diagnosis in patients with TC2BC (15).

Cheng et al. observed that a history of TC was a strong protective factor for $\mathrm{BC}$-specific survival in the TC2BC patients (12). We found that in patients with TC2BC, $\mathrm{BC}$ also had a strong protective effect on the TC-specific survival. In a study by Sandeep et al., the risk of BC after TC increased over time, and there was no evidence that BC treatment increased the risk of TC (16). However, Zhang et al. found that the mean values of TGAb and TPOAb in the TC2BC group were higher than those in the TC group (17). A previous study showed that serum TPOAb and TGAb levels were significantly higher in the BC patients than in the general population (18). Therefore, we believe that the protective effect of second primary $\mathrm{BC}$ on TC might be related to the decrease in the levels of TPOAb and TGAb after BC treatment. However, more research is needed to explore this hypothetical link.

Lei et al. found that TC is a protective factor for the CSS of BC in the BC2TC group and speculated that TSH inhibition therapy is the cause of such protection (19). However, in this study, we found that BC is a risk factor for the CSS of TC in the BC2TC patients. Previous studies have reported that the risk and prognosis of TC may be related to the overexpression of ER and HER2 $(20,21)$, which are common in BC. Therefore, we believe that the adverse effect of $\mathrm{BC}$ on the TC-specific survival may be related to these factors.

Our results showed that both $\mathrm{BC} 2 \mathrm{TC}$ patients and TC2BC patients had higher HRs for OS than TC patients. It is logical that patients with two primary cancers have a lower OS than those who have only one primary cancer. We believe this may be due to the increased risk of complications associated with both types of cancer. Further research is needed to investigate the potential relationship.

There are some limitations of this study. First, the results of this study are heavily influenced by coding biases. This is particularly important because of the long timeframe over which this study was evaluated, during which time several definitions related to TC and BC have evolved. Second, 
in this study, we did not consider the effects of laboratory test results, genetic factors, recurrence, and surgical complications. Third, the study was based on a database rather than clinical data, which may have lowered the level of evidence. Fourth, since only women were selected for the study, it is not clear whether our conclusions would remain valid in men. Finally, this is a retrospective study that is prone to recall bias.

\section{Conclusions}

In conclusion, among women, $\mathrm{BC}$ as the second primary cancer has a significant protective effect on the CSS of TC patients, but when $\mathrm{BC}$ is the first primary cancer, it is a risk factor for worse CSS. BC is a risk factor for the OS of TC patients regardless of whether it is the first or second primary cancer, although it is a stronger risk factor for patients with TC after BC. According to our results, we believe that early diagnosis and treatment of TC in BC survivors should be further strengthened in clinical work, so as to improve the prognosis and survival rate of $\mathrm{BC} 2 \mathrm{TC}$ patients. But further basic and clinical studies are needed to explore more potential relationships between $\mathrm{TC}$ and $\mathrm{BC}$.

\section{Acknowledgments}

Funding: None.

\section{Footnote}

Reporting Checklist: The authors have completed the STROBE reporting checklist. Available at http://dx.doi. org/10.21037/tcr-20-2243

Conflicts of Interest: All authors have completed the ICMJE uniform disclosure form (available at http://dx.doi. org/10.21037/tcr-20-2243). The authors have no conflicts of interest to declare.

Ethical Statement: The authors are accountable for all aspects of the work in ensuring that questions related to the accuracy or integrity of any part of the work are appropriately investigated and resolved. The study was conducted in accordance with the Declaration of Helsinki (as revised in 2013). Since SEER is a publicly available database with anonymized data, ethical approval was not required.

Open Access Statement: This is an Open Access article distributed in accordance with the Creative Commons Attribution-NonCommercial-NoDerivs 4.0 International License (CC BY-NC-ND 4.0), which permits the noncommercial replication and distribution of the article with the strict proviso that no changes or edits are made and the original work is properly cited (including links to both the formal publication through the relevant DOI and the license). See: https://creativecommons.org/licenses/by-nc-nd/4.0/.

\section{References}

1. Siegel RL, Miller KD, Jemal A. Cancer statistics, 2020. CA Cancer J Clin 2020;70:7-30.

2. An JH, Hwangbo Y, Ahn HY, et al. A possible association between thyroid cancer and breast cancer. Thyroid 2015;25:1330-8.

3. Consorti F, Di Tanna G, Milazzo F, et al. Nulliparity enhances the risk of second primary malignancy of the breast in a cohort of women treated for thyroid cancer. World J Surg Oncol 2011;9:88.

4. Subramanian S, Goldstein DP, Parlea L, et al. Second primary malignancy risk in thyroid cancer survivors: a systematic review and meta-analysis. Thyroid 2007;17:1277-88.

5. Kuo JH, Chabot JA, Lee JA. Breast cancer in thyroid cancer survivors: An analysis of the Surveillance, Epidemiology, and End Results-9 database. Surgery 2016;159:23-9.

6. Nielsen SM, White MG, Hong S, et al. The breastthyroid cancer link: a systematic review and meta-analysis. Cancer Epidemiol Biomarkers Prev 2016;25:231-8.

7. Ronckers CM, McCarron P, Ron E. Thyroid cancer and multiple primary tumors in the SEER cancer registries. Int J Cancer 2005;117:281-8.

8. Hung MH, Liu CJ, Teng CJ, et al. Risk of second nonbreast primary cancer in male and female breast cancer patients: a population-based cohort study. PLoS One 2016;11:e0148597.

9. Marcheselli R, Marcheselli L, Cortesi L, et al. Risk of second primary malignancy in breast cancer survivors: a nested population-based case-control study. J Breast Cancer 2015;18:378-85.

10. Huang NS, Chen XX, Wei WJ, et al. Association between breast cancer and thyroid cancer: a study based on 13978 patients with breast cancer. Cancer Med 2018;7:6393-400.

11. Joseph KR, Edirimanne S, Eslick GD. The association between breast cancer and thyroid cancer: a meta-analysis. Breast Cancer Res Treat 2015;152:173-81. 
12. Cheng W, Shen X, Xing M. Decreased breast cancerspecific mortality risk in patients with a history of thyroid cancer. PLoS One 2019;14:e0221093.

13. Li S, Yang J, Shen Y, et al. Clinicopathological features, survival and risk in breast cancer survivors with thyroid cancer: an analysis of the SEER database. BMC Public Health 2019;19:1592.

14. Siołek M, Cybulski C, G sior-Perczak D, et al. CHEK2 mutations and the risk of papillary thyroid cancer. Int $\mathrm{J}$ Cancer $2015 ; 137: 548-52$.

15. Hercbergs A, Mousa SA, Leinung M, et al. Thyroid hormone in the clinic and breast cancer. Horm Cancer 2018;9:139-43.

16. Sandeep TC, Strachan MW, Reynolds RM, et al. Second primary cancers in thyroid cancer patients: a multinational record linkage study. J Clin Endocrinol Metab 2006;91:1819-25.

17. Zhang L, Wu Y, Liu F, et al. Characteristics and survival

Cite this article as: Huang J, Huang Y, Zhou L, Chen S, Chen D, Wei W, Zhang C, Wang M, Zhou W, Zeng W, Liu Z, Guo L. Effect of breast cancer as the first or second primary cancer on the prognosis of women with thyroid cancer: a SEER database analysis. Transl Cancer Res 2020;9(11):69556962. doi: 10.21037/tcr-20-2243 of patients with metachronous or synchronous double primary malignancies: breast and thyroid cancer. Oncotarget 2016;7:52450-9.

18. Muller I, Zhang L, Giani C, et al. The sodium iodide symporter is unlikely to be a thyroid/breast shared antigen. J Endocrinol Invest 2016;39:323-31.

19. Lei K, He X, Yu L, et al. Breast cancer prognosis is better in patients who develop subsequent metachronous thyroid cancer. PLoS One 2019;14:e0215948.

20. Hu ZY, Xiao H, Xiao M, et al. Inducing or preventing subsequent malignancies for breast cancer survivors? Double-edged sword of estrogen receptor and progesterone receptor. Clin Breast Cancer 2018;18:e1149-63.

21. Rebaï M, Kallel I, Hamza F, et al. Association of EGFR and HER2 polymorphisms with risk and clinical features of thyroid cancer. Genet Test Mol Biomarkers 2009;13:779-84. 